\title{
A Hybrid Intelligent Multisensor Positioning Methodology for Reliable Vehicle Navigation
}

\author{
Xu Li, ${ }^{1}$ Qimin Xu, ${ }^{1}$ Chingyao Chan, ${ }^{2}$ Bin Li, ${ }^{3}$ Wei Chen, ${ }^{1}$ and Xianghui Song ${ }^{3}$ \\ ${ }^{1}$ School of Instrument Science and Engineering, Southeast University, Nanjing 210096, China \\ ${ }^{2}$ Institute of Transportation Studies, University of California, Berkeley, CA 94720, USA \\ ${ }^{3}$ Key Laboratory of Technology on Intelligent Transportation Systems, Ministry of Transport, Research Institute of Highway Ministry \\ of Transport, Beijing 100088, China \\ Correspondence should be addressed to Xu Li; lixu.mail@163.com
}

Received 26 March 2015; Revised 4 August 2015; Accepted 24 August 2015

Academic Editor: Hassan Askari

Copyright $(2015 \mathrm{Xu}$ Li et al. This is an open access article distributed under the Creative Commons Attribution License, which permits unrestricted use, distribution, and reproduction in any medium, provided the original work is properly cited.

With the rapid development of intelligent transportation systems worldwide, it becomes more important to realize accurate and reliable vehicle positioning in various environments whether GPS is available or not. This paper proposes a hybrid intelligent multisensor positioning methodology fusing the information from low-cost sensors including GPS, MEMS-based strapdown inertial navigation system (SINS) and electronic compass, and velocity constraint, which can achieve a significant performance improvement over the integration scheme only including GPS and MEMS-based SINS. First, the filter model of SINS aided by multiple sensors is presented in detail and then an improved Kalman filter with sequential measurement-update processing is developed to realize the filtering fusion. Further, a least square support vector machine- (LS SVM-) based intelligent module is designed and augmented with the improved KF to constitute the hybrid positioning system. In case of GPS outages, the LS SVMbased intelligent module trained recently is used to predict the position error to achieve more accurate positioning performance. Finally, the proposed hybrid positioning method is evaluated and compared with traditional methods through real field test data. The experimental results validate the feasibility and effectiveness of the proposed method.

\section{Introduction}

With the rapid development of intelligent transportation systems, global positioning system (GPS) has been widely utilized in many positioning and navigation applications for land vehicles $[1,2]$. The major drawback related to GPS is satellite signal blockage in urban areas, high buildings, and other GPS-denied environments, where its positioning accuracy, reliability, and integrity will be deteriorated $[2,3]$.

In this situation, GPS is usually incorporated with strapdown inertial navigation system (SINS) to achieve accurate and reliable positioning. SINS is a self-contained system that is not affected by external disturbances. However, the standalone SINS solution drifts with time. Through integrating GPS and SINS, one can achieve superior performance over a standalone GPS or SINS [4-6]. SINS consists of a group of inertial measurement units (IMU), that is, three accelerometers and three gyroscopes, which are mounted on a moving platform to measure linear accelerations and angular velocities. Given the initial values of navigation parameters, the measurements from IMU can be processed to determine current position, velocity, and attitude of the moving platform with respect to a certain frame of reference $[7,8]$. Note that the SINS performance depends on its grade. The high-end navigation or tactical grade SINS can maintain proper positioning performance even after a relatively long period of GPS outage. Unfortunately, the high-end SINS is very expensive and thus not suitable for low-cost applications such as those for typical passenger cars. The SINS utilizing microelectromechanical system (MEMS) technology has been developed rapidly in recent years, which provides a viable solution to achieve mass-market applications in land vehicles for its low cost, low power consumption, and small size $[2,7,9]$. However, the MEMS-based SINS sensors may suffer from large noise, bias, and drift errors, which are much more serious than the high-grade sensors $[7,9,10]$. Even 
when GPS outage occurs for a short time, the MEMS-based SINS performance will degrade rapidly.

For the SINS/GPS integration, the Kalman filter (KF) has been widely adopted as the standard optimal estimation tool. However, the limitations of the KF-based SINS/GPS integration approach are the necessity of stochastic modeling of sensor errors, which are difficult to determine especially for MEMS-based gyroscopes and accelerometers, and the need for accurate a priori information of the covariance matrices of the noises associated with both SINS and GPS [8, 10, 11].

Alternatively, artificial intelligence (AI) methods have obtained the popularity in recent years due to their abilities to solve the problem of nonlinearity and uncertainty. These AIbased approaches include Random Forest Regression (RFS) [12], Multilayer Perceptron Neural Networks (MLPNNs) [13], Adaptive Neurofuzzy Inference System (ANFIS) [10, 14, 15], and Radial Basis Function Neural Networks (RBFNNs) [8, 16-18].

Generally, although the AI-based methods above perform well for the navigation or tactical grade SINS, they have shown a very limited success when applied to a MEMS-based SINS/GPS navigation system [12]. The main reason is due to the high noise level and bias instability of MEMS-based inertial sensors, which increases the nonlinear complexity of the input/output functional relationship being modeled and limits the AI generalization ability that in turn affects its prediction accuracy. To overcome the standalone MEMSbased SINS limitation when GPS outage occurs, an effective and viable solution is to fuse other sensors, especially lowcost ones in land vehicle applications $[2,9]$. Through fusing more information from other sensors or sources, one can take advantage of the complementary properties of different sensors and thus enhance the accuracy and reliability of the positioning system. For instance, if the yaw angle information from low-cost electronic compass is fused into the system during GPS outages, the accumulative error of yaw angle caused by the integration of low-performance MEMS gyroscope will be eliminated, and then the horizontal positioning performance will be improved significantly because it is affected largely by the azimuth accuracy.

In this paper, a hybrid intelligent multisensor positioning methodology is proposed, which integrates the information from low-cost sensors including GPS, MEMS-based IMU and electronic compass, and velocity constraint. An improved Kalman filter (KF) with sequential measurement-update processing is first developed to fuse different information. Further, a least square support vector machine- (LS SVM-) based intelligent module is designed and augmented with the improved KF to form the hybrid positioning system. The hybrid positioning system has two modes of operation, that is, the update and prediction modes, which are dependent on the situation of GPS outage. The novel aspects of this paper can be summarized as follows:

(1) Instead of only integrating SINS and GPS as presented in much AI-based literature, the proposed hybrid positioning methodology has the capability of fusing more information from other low-cost sensors or sources through combining the improved KF with the LS SVM-based intelligent algorithm. The distinct advantage is that, without adding much cost, the proposed methodology can significantly improve the positioning performance.

(2) The proposed methodology exhibits strong generalization ability even when the GPS outage duration exceeds the length of the sliding window designed for training the LS VSM, which has been verified by real field tests. To the authors' knowledge, the performance of the prediction stability is seldom evaluated and discussed in such cases in the existing AI-based literature.

This paper is organized as follows. Section 2 gives an overview of the proposed hybrid positioning methodology for reliable land vehicle navigation. The improved Kalman filter is presented in detail in Section 3. Section 4 describes the design of LS SVM-based intelligent module. Field tests and experimental results are provided in Section 5. Section 6 is devoted to the conclusion.

\section{Proposed Hybrid Positioning Methodology}

To achieve accurate and reliable positioning performance for land vehicles, a hybrid positioning methodology of augmenting improved KF with LS SVM is proposed, which fuses the information from low-cost sensors including GPS, MEMSbased IMU and electronic compass, and lateral velocity constraint. The augmented system comprises two modes of operation, that is, the update mode and the prediction mode.

When GPS is available, the system operates in the update (i.e., training) mode, as shown in Figure 1(a). It is noted that the Kalman filter is improved here to work with sequential measurement-update processing, which is different from the centralized processing as utilized in the common KF. The measurement update 1 is associated with the measurements of electronic compass and "virtual" lateral velocity (i.e., $\widetilde{V}_{Y_{b}}=$ 0 ), while the measurement update 2 is associated with GPS measurements. In actual implementation, two KF processing modules are executed. For the first module, simulated successive $w$-s GPS signal outages are applied ( $w$ denotes the duration of simulated GPS outage) and the position error estimated after the measurement update 1 can be attainable. For the second module, the GPS measurements are available at all times and thus the position error estimated after two sequential measurement updates 1 and 2 is always obtained. The difference between two position errors above at each epoch is then transferred to the LS SVM-based intelligent module as its desired output during the training process. Meanwhile, the time elapsed since GPS is unavailable and the IMU output act as the inputs of LS VSM at the same epoch. Besides, considering the balance between desired accuracy and computation efficiency, a $40 \mathrm{~s}$ length sliding window of input/output data pattern (i.e., the window size $w=40$ ) is chosen for the adaptive optimization of LS SVM training algorithm. 


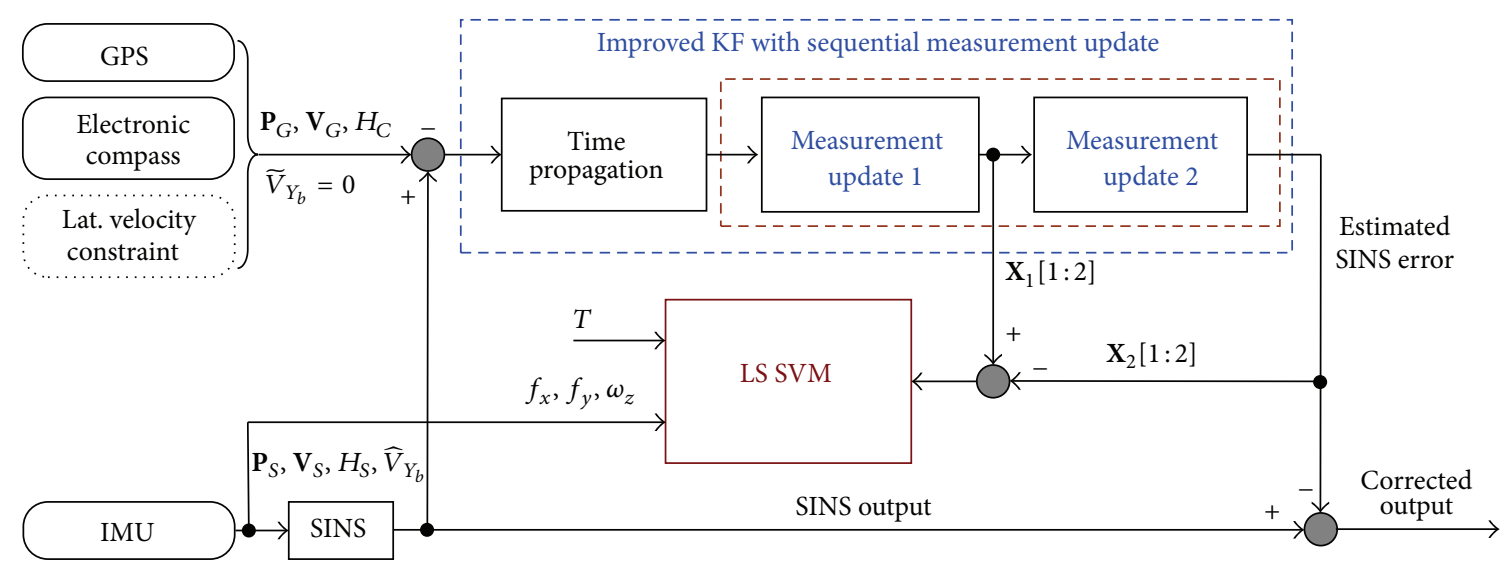

(a)

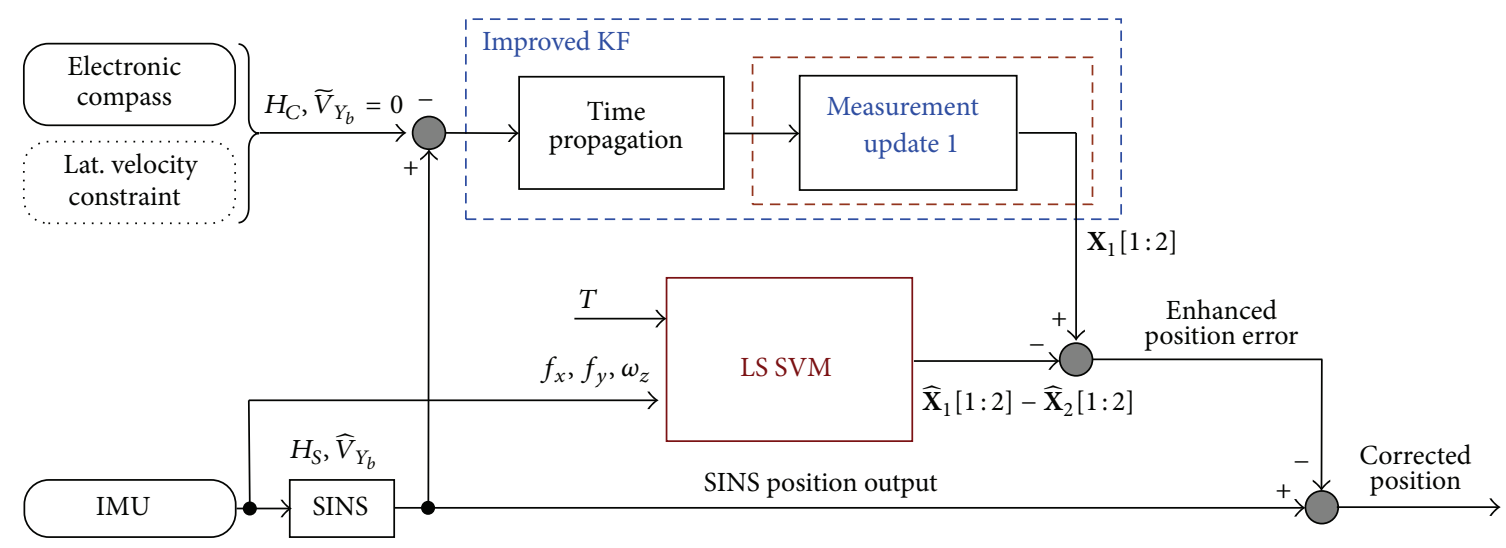

(b)

Figure 1: Proposed hybrid positioning methodology. (a) The update mode; (b) the prediction mode.

Whenever the GPS signal is lost due to high buildings, tunnels, or other factors, the measurement update 2 cannot be executed but the measurement update 1 can still be carried out. In this case, the system operation automatically switches to the prediction mode shown in Figure 1(b), where the LS SVM-based intelligent module is utilized to predict the difference between the position error estimated after the measurement update 1 and that estimated after sequential measurement updates 1 and 2. Based on the time elapsed from the moment of GPS outage and the IMU output, the LS SVM uses the latest LS VSM parameters updated just before losing the satellite signals to predict the difference between two position errors mentioned above. Then, utilizing the available position error estimated after the measurement update 1 , the position error contained in SINS can be acquired. Finally, such enhanced position error can be removed from the SINS position output to obtain more precise and reliable positioning solution for land vehicles.

\section{Improved Kalman Filter}

In this section, we describe the construction of the improved Kalman filter that is adopted for this work.
3.1. System State Equation. According to SINS mechanization, the dynamic error model of the SINS navigation parameters (i.e., position, velocity, and attitude) can be described as $[19,20]$

$$
\begin{aligned}
\delta \dot{\mathbf{P}}= & \mathbf{D}_{1} \delta \mathbf{P}+\mathbf{D}_{2} \delta \mathbf{V}^{n}, \\
\delta \dot{\mathbf{V}}^{n}= & \mathbf{f}^{n} \times \boldsymbol{\psi}^{n}-\left(2 \delta \boldsymbol{\omega}_{i e}^{n}+\delta \boldsymbol{\omega}_{e n}^{n}\right) \times \mathbf{V}^{n}-\left(2 \boldsymbol{\omega}_{i e}^{n}+\boldsymbol{\omega}_{e n}^{n}\right) \\
& \times \delta \mathbf{V}^{n}+\mathbf{C}_{b}^{n} \delta \mathbf{f}^{b}, \\
\dot{\psi}^{n}= & \delta \boldsymbol{\omega}_{i e}^{n}+\delta \boldsymbol{\omega}_{e n}^{n}-\left(\boldsymbol{\omega}_{i e}^{n}+\boldsymbol{\omega}_{e n}^{n}\right) \times \psi^{n}-\mathbf{C}_{b}^{n} \delta \boldsymbol{\omega}_{i b}^{b},
\end{aligned}
$$

where $\delta \mathbf{P}=\left[\begin{array}{lll}\delta L & \delta \lambda & \delta h\end{array}\right]^{T}$ is the position error vector (latitude, longitude, and height), $\delta \mathbf{V}^{n}=\left[\begin{array}{llll}\delta V_{E} & \delta V_{N} & \delta V_{U}\end{array}\right]^{T}$ is the velocity error vector (east, north, and up), $\psi^{n}=$ $\left[\begin{array}{lll}\psi_{E} & \psi_{N} & \psi_{U}\end{array}\right]^{T}$ is the attitude error vector, $\mathbf{C}_{b}^{n}$ is the strapdown matrix, $\boldsymbol{\omega}_{i e}^{n}$ is the angular rate vector of the rotation of the Earth relative to the inertial frame, $\boldsymbol{\omega}_{e n}^{n}$ is the angular rate vector of the rotation of the navigation frame relative to the Earth, $\mathbf{f}^{n}$ and $\mathbf{V}^{n}$ are specific force and velocity vectors in the navigation frame, respectively, $\delta \mathbf{f}^{b}$ and $\delta \boldsymbol{\omega}_{i b}^{b}$ are accelerometer bias and gyro drift vectors in the body frame, respectively, and $\mathbf{D}_{1}$ and $\mathbf{D}_{2}$ are two $3 \times 3$ matrices whose nonzero elements are functions of the vehicle's latitude $(L)$ and height $(h)$. 
In addition, considering inertial sensor residuals, the state vector of Kalman filter (KF) is composed of fifteen states, which include nine navigation parameter errors above (i.e., position, velocity, and attitude), three accelerometer biases, and three gyro drifts:

$$
\mathbf{X}=\left[\begin{array}{lllllllllllllll}
\delta L & \delta \lambda & \delta h & \delta V_{E} & \delta V_{N} & \delta V_{U} & \psi_{E} & \psi_{N} & \psi_{U} & \nabla_{b x} & \nabla_{b y} & \nabla_{b z} & \varepsilon_{b x} & \varepsilon_{b y} & \varepsilon_{b z}
\end{array}\right]^{T}
$$

where $\nabla_{b x}, \nabla_{b y}$, and $\nabla_{b z}$ denote three accelerometer constant biases in the body frame, while $\varepsilon_{b x}, \varepsilon_{b y}$, and $\varepsilon_{b z}$ are three gyro constant drifts in the body frame.

Based on (1) and inertial sensor residual model $[19,20]$, the continuous-time system state equation of KF can be expressed as

$$
\dot{\mathbf{X}}(t)=\mathbf{F}(t) \cdot \mathbf{X}(t)+\mathbf{G}(t) \cdot \mathbf{W}(t)
$$

where $\mathbf{G}$ is the system noise input matrix, $\mathbf{W}$ is the system noise vector, and $\mathbf{F}$ is the system state propagation matrix. The definitions of $\mathbf{F}, \mathbf{G}$, and $\mathbf{W}$ are given in Appendix.

According to the method described in [20], (3) is discretized. The discrete-time system state equation is

$$
\mathbf{X}(k+1)=\boldsymbol{\Phi}(k+1, k) \mathbf{X}(k)+\Gamma(k) \mathbf{W}(k),
$$

where $k$ refers to the discrete-time step, $\mathbf{X}(k)$ is the discretetime state vector, $\Phi(k+1, k)$ is the discrete-time state transition matrix, $\Gamma(k)$ is the discrete system noise input matrix, and $\mathbf{W}(k)$ is the discrete system noise with covariance matrix $\mathbf{Q}(k)$. How to determine $\boldsymbol{\Phi}(k+1, k), \boldsymbol{\Gamma}(k)$, and $\mathbf{W}(k)$ is also given in Appendix.

3.2. Measurement Model. According to Figure 1, the KF measurements consist of three parts, that is, the measurement of electronic compass, the lateral velocity constraint, and the GPS measurements.

First, the measurement model about electronic compassaided SINS can be built up according to the coordinate transformation theory [21]. Here, let $H_{C}$ represent the vehicle's yaw angle measured by electronic compass and it can be described as

$$
H_{C}=H_{t}-n_{H_{C}}
$$

where $H_{t}$ denotes the true yaw angle and $n_{H_{C}}$ is the compass measurement Gauss white noise. On the other hand, we suppose that $H_{S}$ represents the yaw angle calculated by SINS and $\delta H_{S}$ denotes the subtraction between $H_{S}$ and $H_{t}$ :

$$
H_{S}=H_{t}+\delta H_{S}
$$

According to the inertial navigation principle, the relationship between the ideal strapdown matrix $\mathbf{C}_{b}^{n}$ and the true attitude angles is

$$
\mathbf{C}_{b}^{n}=\left[\begin{array}{ccc}
\cos H_{t} \cos P_{t} & \cos H_{t} \sin P_{t} \sin R_{t}-\sin H_{t} \cos R_{t} & \cos H_{t} \sin P_{t} \cos R_{t}+\sin H_{t} \sin R_{t} \\
\sin H_{t} \cos P_{t} & \sin H_{t} \sin P_{t} \sin R_{t}+\cos H_{t} \cos R_{t} & \sin H_{t} \sin P_{t} \cos R_{t}-\cos H_{t} \sin R_{t} \\
-\sin P_{t} & \cos P_{t} \sin R_{t} & \cos P_{t} \cos R_{t}
\end{array}\right]
$$

where $P_{t}$ and $R_{t}$ are true pitch and roll angles of the vehicle, respectively.
Correspondingly, the calculated strapdown matrix $\mathbf{C}_{b}^{p}$ (the symbol $p$ denotes the SINS's calculated platform frame) can be described as

$$
\mathbf{C}_{b}^{p}=\left[\begin{array}{ccc}
\cos H_{S} \cos P_{S} & \cos H_{S} \sin P_{S} \sin R_{S}-\sin H_{S} \cos R_{S} & \cos H_{S} \sin P_{S} \cos R_{S}+\sin H_{S} \sin R_{S} \\
\sin H_{S} \cos P_{S} & \sin H_{S} \sin P_{S} \sin R_{S}+\cos H_{S} \cos R_{S} & \sin H_{S} \sin P_{S} \cos R_{S}-\cos H_{S} \sin R_{S} \\
-\sin P_{S} & \cos P_{S} \sin R_{S} & \cos P_{S} \cos R_{S}
\end{array}\right],
$$


where $H_{S}, P_{S}$, and $R_{S}$ are the yaw, pitch, and roll angles of the vehicle calculated by SINS, respectively.

The relationship between $\mathbf{C}_{b}^{p}$ and $\mathbf{C}_{b}^{n}$ is [19]

$$
\mathbf{C}_{b}^{p}=\left(\mathbf{I}-\psi^{n} \times\right) \mathbf{C}_{b}^{n}
$$

where $\psi^{n} \times$ is the skew-symmetric form of the vector $\psi^{n}$. Expanding (9) in terms of each corresponding element, then we obtain two useful equations; that is,

$$
\begin{aligned}
& \mathbf{C}_{b}^{p}(1,1)=\mathbf{C}_{b}^{n}(1,1)+\mathbf{C}_{b}^{n}(2,1) \psi_{U}-\mathbf{C}_{b}^{n}(3,1) \psi_{N}, \\
& \mathbf{C}_{b}^{p}(2,1)=-\mathbf{C}_{b}^{n}(1,1) \psi_{U}+\mathbf{C}_{b}^{n}(2,1)+\mathbf{C}_{b}^{n}(3,1) \psi_{E},
\end{aligned}
$$

where $\mathbf{C}_{b}^{p}(i, j)$ denotes the $i$-row and $j$-column element of $\mathbf{C}_{b}^{p}$ $(i=1,2,3$ and $j=1,2,3)$. Further, based on (8), the following can be gotten:

$$
\begin{aligned}
H_{S} & =\arctan \left[\frac{\mathbf{C}_{b}^{p}(2,1)}{\mathbf{C}_{b}^{p}(1,1)}\right] \\
& =\arctan \left[\frac{\mathbf{C}_{b}^{n}(2,1)-\mathbf{C}_{b}^{n}(1,1) \psi_{U}+\mathbf{C}_{b}^{n}(3,1) \psi_{E}}{\mathbf{C}_{b}^{n}(1,1)+\mathbf{C}_{b}^{n}(2,1) \psi_{U}-\mathbf{C}_{b}^{n}(3,1) \psi_{N}}\right],
\end{aligned}
$$

where $\mathbf{C}_{b}^{n}(i, j)$ denotes the $i$-row and $j$-column element of $\mathbf{C}_{b}^{n}$ $(i=1,2,3$ and $j=1,2,3)$.

Perturbing (11), the yaw-angle error equation can be obtained as

$$
\delta H_{S}=\frac{\partial H_{S}}{\partial \psi_{E}} \psi_{E}+\frac{\partial H_{S}}{\partial \psi_{N}} \psi_{N}+\frac{\partial H_{S}}{\partial \psi_{U}} \psi_{U},
$$

where $\partial H_{S} / \partial \psi_{E}=\mathbf{C}_{b}^{n}(1,1) \mathbf{C}_{b}^{n}(3,1) /\left(\left[\mathbf{C}_{b}^{n}(1,1)\right]^{2}+\left[\mathbf{C}_{b}^{n}(2,1)\right]^{2}\right)$, $\partial H_{S} / \partial \psi_{N}=\mathbf{C}_{b}^{n}(2,1) \mathbf{C}_{b}^{n}(3,1) /\left(\left[\mathbf{C}_{b}^{n}(1,1)\right]^{2}+\left[\mathbf{C}_{b}^{n}(2,1)\right]^{2}\right)$, and $\partial H_{S} / \partial \psi_{U}=-1$.

Subtracting (5) from (6) and utilizing (12), we can get

$$
H_{S}-H_{C}=\frac{\partial H_{S}}{\partial \psi_{E}} \psi_{E}+\frac{\partial H_{S}}{\partial \psi_{N}} \psi_{N}+\frac{\partial H_{S}}{\partial \psi_{U}} \psi_{U}+n_{H_{C}} .
$$

Then, we will establish the measurement equation about lateral velocity constraint. It is well-known that, in general driving conditions, the vehicle does not slip or does slip very little. Thus, we can make the reasonable assumption that, for land vehicle positioning applications, the virtual measurement of lateral velocity is zero (i.e., lateral velocity constraint) [22]:

$$
\widetilde{V}_{Y_{b}}=0 \text {, }
$$

where $\widetilde{V}_{Y_{b}}$ denotes the virtually measured lateral velocity of the vehicle in the body frame. Besides, according to the coordinate transformation theory, we have

$$
\widetilde{V}_{Y_{b}}=\left[\mathbf{C}_{n}^{b}\right]_{2,1: 3} \mathbf{V}^{n}-n_{V_{Y_{b}}},
$$

where $\left[\mathbf{C}_{n}^{b}\right]_{2,1: 3}$ denotes a $1 \times 3$ new matrix composed of the 3 elements lying in the second row of $\mathbf{C}_{n}^{b} \cdot \mathbf{V}^{n}=\left[\begin{array}{llll}V_{E} & V_{N} & V_{U}\end{array}\right]^{T}$ is the true velocity vector of the vehicle in the navigation frame. $n_{V_{Y_{b}}}$ is the virtual measurement noise that represents the error in the assumption above.

Denoting the vehicle's velocity vector calculated by SINS in the navigation frame as $\widehat{\mathbf{V}}^{n}=\left[\begin{array}{lll}\widehat{V}_{E} & \widehat{V}_{N} & \widehat{V}_{U}\end{array}\right]^{T}$, the following equation can be yielded:

$$
\widehat{V}_{Y_{b}}=\left[\mathbf{C}_{p}^{b}\right]_{2,1: 3} \cdot \widehat{\mathbf{V}}^{n}=\left[\mathbf{C}_{p}^{b}\right]_{2,1: 3} \cdot\left(\mathbf{V}^{n}+\delta \mathbf{V}^{n}\right),
$$

where $\widehat{V}_{Y_{b}}$ represents the lateral velocity calculated by SINS in the body frame and $\delta \mathbf{V}^{n}=\left[\begin{array}{lll}\delta V_{E} & \delta V_{N} & \delta V_{U}\end{array}\right]^{T}$ is the difference between $\widehat{\mathbf{V}}^{n}$ and $\mathbf{V}^{n}$. $\left[\mathbf{C}_{p}^{b}\right]_{2,1: 3}$ is a $1 \times 3$ new matrix composed of the 3 elements lying in the second row of $\mathbf{C}_{p}^{b}$.

Considering $\mathbf{C}_{p}^{b}=\mathbf{C}_{n}^{b}\left[\mathbf{I}+\psi^{n} \times\right]$ and rearranging (16), we can obtain

$$
\begin{aligned}
\widehat{V}_{Y_{b}}= & {\left[\mathbf{C}_{n}^{b}\right]_{2,1: 3} \mathbf{V}^{n}+\left[\mathbf{C}_{n}^{b}\right]_{2,1: 3} \delta \mathbf{V}^{n} } \\
& +\left[\mathbf{C}_{n}^{b}\left(\psi^{n} \times\right)\right]_{2,1: 3} \mathbf{V}^{n}+\left[\mathbf{C}_{n}^{b}\left(\boldsymbol{\psi}^{n} \times\right)\right]_{2,1: 3} \delta \mathbf{V}^{n}
\end{aligned}
$$

Note that the last item of (17) is a second-order small quantity and can be neglected. Based on the skew-symmetric matrix features, we subtract (15) from (17) and then obtain

$$
\begin{aligned}
\widehat{V}_{Y_{b}}-\widetilde{V}_{Y_{b}} & =\widehat{V}_{Y_{b}}-0 \\
& =\left[\mathbf{C}_{n}^{b}\right]_{2,1: 3} \delta \mathbf{V}^{n}-\left[\mathbf{C}_{n}^{b}\left(\mathbf{V}^{n} \times\right)\right]_{2,1: 3} \psi^{n}+n_{V_{Y_{b}}},
\end{aligned}
$$

where $\mathbf{V}^{n} \times$ denotes the skew-symmetric matrix formed by the vector $\mathbf{V}^{n}$.

For convenient discussion later, combining (13) and (18), the measurement model about SINS aided by both electronic compass and lateral velocity constraint can be obtained:

$$
\mathbf{Z}_{1}=\mathbf{H}_{1} \cdot \mathbf{X}+\mathbf{V}_{1}
$$

where $\mathbf{Z}_{1}=\left[H_{S}-H_{C} \widehat{V}_{Y_{b}}-0\right]^{T}$ is the measurement information. $\mathbf{H}_{1}$ is the corresponding observation matrix, which can be determined according to (13) and (18). $\mathbf{V}_{1}$ is the measurement noise vector with the covariance matrix $\mathbf{R}_{1}$.

Finally, the measurement model about GPS-aided SINS can be easily built by subtracting the GPS measurement information from the SINS's corresponding output information. When GPS is available, it can provide accurate position and velocity information for the vehicle, that is, $\mathbf{P}_{G}$ and $\mathbf{V}_{G}$ shown in Figure 1(a). Thus, the measurement model of GPS-aided SINS can be expressed as

$$
\mathbf{Z}_{2}=\mathbf{H}_{2} \cdot \mathbf{X}+\mathbf{V}_{2}
$$

where $\mathbf{Z}_{2}$ and $\mathbf{H}_{2}$ are GPS measurement information and observation matrix, respectively, and they are discussed in detail in [19]. $\mathbf{V}_{2}$ is the corresponding measurement noise vector with the covariance matrix $\mathbf{R}_{2}$. 
Combining (19) and (20), the overall measurement equation of the KF can be written as

$$
\mathbf{Z}=\mathbf{H} \cdot \mathbf{X}+\mathbf{V}
$$

where $\mathbf{Z}(k)=\left[\begin{array}{ll}\mathbf{Z}_{1}^{T} & \mathbf{Z}_{2}^{T}\end{array}\right]^{T}$ is the measurement vector of the KF. $\mathbf{H}=\left[\begin{array}{ll}\mathbf{H}_{1}^{T} & \mathbf{H}_{2}^{T}\end{array}\right]^{T}$ is the observation matrix. $\mathbf{V}(k)=\left[\begin{array}{ll}\mathbf{V}_{1}^{T} & \mathbf{V}_{2}^{T}\end{array}\right]^{T}$ is the observation noise vector with the covariance matrix $\mathbf{R}=$ $\operatorname{diag}\left(\mathbf{R}_{\mathbf{1}}, \mathbf{R}_{2}\right)$.

3.3. Improved Kalman Filtering Algorithm. For the model described by the system state equation (4) and the measurement equation (21), we can execute the filtering fusion based on the standard KF algorithm, which comprises two phases, that is, time propagation and measurement update.

(1) Time Propagation. Utilizing (4), the equation for state prediction is

$$
\mathbf{X}(k+1, k)=\Phi(k+1, k) \mathbf{X}(k) .
$$

The covariance of the state prediction $\mathbf{P}(k+1, k)$ is

$$
\begin{aligned}
\mathbf{P}(k+1, k)= & \boldsymbol{\Phi}(k+1, k) \mathbf{P}(k) \boldsymbol{\Phi}(k+1, k)^{T} \\
& +\boldsymbol{\Gamma}(k) \mathbf{Q}(k) \boldsymbol{\Gamma}(k)^{T} .
\end{aligned}
$$

(2) Measurement Update. The Kalman gain can be calculated as

$$
\begin{aligned}
& \mathbf{K}(k+1) \\
& =\mathbf{P}(k+1, k) \cdot \mathbf{H}^{T}(k+1) \\
& \quad \cdot\left[\mathbf{H}(k+1) \mathbf{P}(k+1, k) \mathbf{H}^{T}(k+1)+\mathbf{R}(k+1)\right]^{-1} .
\end{aligned}
$$

The estimated state vector after measurement update is

$$
\begin{aligned}
\mathbf{X}(k+1) \\
=\mathbf{X}(k+1, k) \\
\quad+\mathbf{K}(k+1)[\mathbf{Z}(k+1)-\mathbf{H}(k+1) \cdot \mathbf{X}(k+1, k)]
\end{aligned}
$$

and the updated covariance is

$$
\mathbf{P}(k+1)=[\mathbf{I}-\mathbf{K}(k+1) \mathbf{H}(k+1)] \mathbf{P}(k+1, k) .
$$

From (24)-(26), it can be seen that traditional measurement update processes all the measurement information in a centralized mode. The measurement-update results associated with different observation vectors (e.g., $\mathbf{Z}_{1}$ and $\mathbf{Z}$ here) cannot be acquired in such centralized mode, and thus it is difficult to realize the mechanization proposed in Section 2. Besides, the centralized processing mode has another limitation that it is only suitable for the situation when GPS is available. When the GPS signal is missing, the GPS measurements cannot be obtained and its corresponding measurement update should be excluded, whereas the measurement update associated with electronic compass and lateral velocity constraint should still be executed. To overcome the limitations of traditional measurement-update processing, a sequential measurement-update processing algorithm is developed here.

To realize sequential measurement-update processing for different observation sources, that is, $\mathbf{Z}_{1}$ and $\mathbf{Z}_{2}$, we divide the matrices involved in the overall measurement update into two corresponding subblocks before the measurement update is executed, which is actually equivalent to scalar measurement processing discussed in $[23,24]$. Namely, let

$$
\begin{aligned}
\mathbf{P}_{0} & =\mathbf{P}(k+1, k), \\
\mathbf{X}_{0} & =\mathbf{X}(k+1, k), \\
\mathbf{H}(k) & =\left[\begin{array}{l}
\mathbf{H}_{1} \\
\mathbf{H}_{2}
\end{array}\right], \\
\mathbf{Z}(k) & =\left[\begin{array}{l}
\mathbf{Z}_{1} \\
\mathbf{Z}_{2}
\end{array}\right], \\
\mathbf{R} & =\left[\begin{array}{ll}
\mathbf{R}_{1} & 0 \\
\mathbf{0} & \mathbf{R}_{2}
\end{array}\right] .
\end{aligned}
$$

Based on the division above, the measurement update is executed in a sequential order. That is, the measurement update about $\mathbf{Z}_{1}$ is performed first, and then the measurement update related to GPS (i.e., $\mathbf{Z}_{2}$ ) is performed. Specifically, although the time propagation is still executed according to (22)-(23), the measurement update is sequentially executed according to the following two steps.

Step 1. First, execute the measurement update about $\mathbf{Z}_{1}$,

$$
\begin{aligned}
& \mathbf{K}_{1}(k+1)=\mathbf{P}_{0}(k+1, k) \cdot \mathbf{H}_{1}^{T}(k+1) \\
& \quad \cdot\left[\mathbf{H}_{1}(k+1) \mathbf{P}_{0}(k+1, k) \mathbf{H}_{1}^{T}(k+1)\right. \\
& \left.\quad+\mathbf{R}_{1}(k+1)\right]^{-1}, \\
& \mathbf{X}_{1}(k+1)=\mathbf{X}_{0}(k+1, k)+\mathbf{K}_{1}(k+1)\left[\mathbf{Z}_{1}(k+1)\right. \\
& \left.\quad-\mathbf{H}_{1}(k+1) \cdot \mathbf{X}_{0}(k+1, k)\right] \\
& \mathbf{P}_{1}(k+1)=\left[\mathbf{I}-\mathbf{K}_{1}(k+1) \mathbf{H}_{1}(k+1)\right] \mathbf{P}_{0}(k+1, k) .
\end{aligned}
$$

Step 2. Further, execute the measurement update about $\mathbf{Z}_{2}$,

$$
\begin{aligned}
& \mathbf{K}_{2}(k+1) \\
& =\mathbf{P}_{1}(k+1) \cdot \mathbf{H}_{2}^{T}(k+1) \\
& \quad \cdot\left[\mathbf{H}_{2}(k+1) \mathbf{P}_{1}(k+1) \mathbf{H}_{2}^{T}(k+1)+\mathbf{R}_{2}(k+1)\right]^{-1} \\
& \mathbf{X}_{2}(k+1) \\
& =\mathbf{X}_{1}(k+1) \\
& \quad+\mathbf{K}_{2}(k+1)\left[\mathbf{Z}_{2}(k+1)-\mathbf{H}_{2}(k) \cdot \mathbf{X}_{1}(k+1)\right] \\
& \mathbf{P}_{2}(k+1)=\left[\mathbf{I}-\mathbf{K}_{2}(k+1) \mathbf{H}_{2}(k+1)\right] \mathbf{P}_{1}(k+1) .
\end{aligned}
$$


In (28)-(29), $\mathbf{X}_{1}(k+1)$ and $\mathbf{P}_{1}(k+1)$ are the estimated state vector and covariance matrix after the measurement update about $\mathbf{Z}_{1}$, respectively, whereas $\mathbf{X}_{2}(k+1)$ and $\mathbf{P}_{2}(k+1)$ are the estimated state vector and covariance matrix after the measurement update about $\mathbf{Z}_{2}$, respectively. Besides, $\mathbf{X}_{2}(k+1)$ and $\mathbf{P}_{2}(k+1)$ also act as the initial values of time propagation of the next time $k+1$; that is, $\mathbf{X}(k+1)=\mathbf{X}_{2}(k+1)$ and $P(k+1)=\mathbf{P}_{2}(k+1)$.

Note that the improved measurement-update processing described above is corresponding to the situation when GPS is available. In such situation, the measurement updates about $\mathbf{Z}_{1}$ and $\mathbf{Z}_{2}$ are executed sequentially according to Steps 1 and 2 above. Thus, $\mathbf{X}_{1}$ and $\mathbf{X}_{2}$ can easily be obtained, which are utilized for the update mode as shown in Figure 1(a). When the GPS signal is lost, only the observation information about $\mathbf{Z}_{1}$ is executed and so only the measurement-update algorithm described by Step 1 is executed. In this case, $\mathbf{X}_{1}$ can still be acquired, which is utilized for the prediction mode as illustrated in Figure 1(b). Therefore, the improved measurement-update processing provides an effective and feasible solution for the proposed methodology.

\section{Design of LS SVM-Based Intelligent Module}

The least square support vector machine (LS SVM) is a statistical learning theory which adopts a least squares linear system as the loss function instead of the quadratic program $[25,26]$. LS SVM is based on the principle of structure risk minimization, which minimizes an upper bound on the generalization error, and thus its generalization performance is superior to that of the common AI methods adopting empirical risk minimization $[27,28]$. The excellent generalization ability of LS SVM has been verified to be very effective to solve both regression and classification problems, and it has also been successfully applied for solving many engineering problems.

4.1. LS SVM Algorithm. Consider a given training set of $N$ data points $\left\{\mathbf{x}_{k}, y_{k}\right\}_{k=1}^{N}$ with input data $\mathbf{x}_{k} \in \mathbf{R}^{n}$ and output $y_{k} \in r$ where $\mathbf{R}^{n}$ is the $n$-dimensional vector space and $r$ is the one-dimensional vector space. In the feature space, the LS SVM model takes the form

$$
f(\mathbf{x})=\boldsymbol{\omega}^{T} \varphi(\mathbf{x})+b,
$$

where $\boldsymbol{\omega} \in \mathbf{R}^{n_{h}}$ is an adjustable weight vector, $\varphi(\cdot): \mathbf{R}^{n} \rightarrow$ $\mathbf{R}^{n_{h}}$ is the nonlinear function that maps the input data into a higher dimensional feature space, and $b \in r$ is the scalar threshold.

In the LS SVM for function estimation, the following optimization problem is formulated:

$$
\begin{array}{ll}
\min & J(\boldsymbol{\omega}, b, e)=\frac{1}{2} \boldsymbol{\omega}^{T} \boldsymbol{\omega}+\frac{1}{2} \gamma \sum_{k=1}^{N} e_{k}^{2} \\
\text { s.t. } & y_{k}=\boldsymbol{\omega}^{T} \varphi\left(\mathbf{x}_{k}\right)+b+e_{k}, \quad k=1, \ldots, N,
\end{array}
$$

where $e_{k}$ is the error variable and $\gamma \geq 0$ is a regularization constant. Smaller $\gamma$ can avoid the overfitting problem in cases where data are noisy.

For (31), the Lagrangian is given by

$$
\begin{aligned}
L_{\mathrm{LS} \mathrm{SVM}}= & \frac{1}{2} \boldsymbol{\omega}^{T} \boldsymbol{\omega}+\frac{1}{2} \gamma \sum_{k=1}^{N} e_{k}^{2} \\
& -\sum_{k=1}^{N} \alpha_{k}\left\{\boldsymbol{\omega}^{T} \varphi\left(\mathbf{x}_{k}\right)+b+e_{k}-y_{k}\right\},
\end{aligned}
$$

where $\alpha_{k} \in r(k=1, \ldots, N)$ are the Lagrange multipliers. Then, the conditions for optimality are

$$
\begin{array}{rl}
\frac{\partial L_{\mathrm{LS} \mathrm{SVM}}}{\partial \boldsymbol{\omega}} & =0 \longrightarrow \boldsymbol{\omega}=\sum_{k=1}^{N} \alpha_{k} \varphi\left(\mathbf{x}_{k}\right) \\
\frac{\partial L_{\mathrm{LS} \mathrm{SVM}}}{\partial b} & =0 \longrightarrow \sum_{k=1}^{N} \alpha_{k}=0 \\
\frac{\partial L_{\mathrm{LS} \mathrm{SVM}}}{\partial e_{k}} & =0 \longrightarrow \alpha_{k}=\gamma e_{k}, \quad k=1, \ldots, N \\
\frac{\partial L_{\mathrm{LS} \mathrm{SVM}}}{\partial \alpha_{k}} & =0 \longrightarrow \boldsymbol{\omega}^{T} \varphi\left(\mathbf{x}_{k}\right)+b+e_{k}-y_{k}=0, \\
k & k=1, \ldots, N .
\end{array}
$$

Eliminating $\boldsymbol{\omega}$ and $e_{k}$ will yield a linear system instead of a quadratic programming problem

$$
\left[\begin{array}{cc}
0 & \mathbf{1}_{N}^{T} \\
\mathbf{1}_{N} & \boldsymbol{\Omega}+\frac{\mathbf{I}_{N}}{\gamma}
\end{array}\right]\left[\begin{array}{l}
b \\
\boldsymbol{\alpha}
\end{array}\right]=\left[\begin{array}{l}
0 \\
\mathbf{Y}
\end{array}\right],
$$

where $\mathbf{Y}=\left[y_{1}, \ldots, y_{N}\right]^{T}, \mathbf{1}_{N}=[1, \ldots, 1]^{T}$, and $\boldsymbol{\alpha}=\left[\alpha_{1}, \ldots\right.$, $\left.\alpha_{N}\right]^{T} . \mathbf{I}_{N}$ is $N \times N$ identity matrix. $\mathbf{\Omega} \in \mathbf{R}^{N \times N}$ is the kernel matrix defined by

$$
\boldsymbol{\Omega}_{i j}=\varphi\left(\mathbf{x}_{i}\right)^{T} \varphi\left(\mathbf{x}_{j}\right)=K\left(\mathbf{x}_{i}, \mathbf{x}_{j}\right), \quad i, j=1, \ldots, N .
$$

The radial basis function (RBF) has been used here as the kernel function $K(\cdot, \cdot)$, which is given by

$$
K\left(\mathbf{x}, \mathbf{x}_{k}\right)=\exp \left\{-\frac{\left\|\mathbf{x}-\mathbf{x}_{k}\right\|_{2}^{2}}{2 \sigma^{2}}\right\}, \quad k=1, \ldots, N,
$$

where $\left\|\mathbf{x}-\mathbf{x}_{k}\right\|_{2}^{2}$ is the squared Euclidean distance between the two feature vectors and $\sigma$ is the width of RBF.

Further, the result of the LS SVM model for function estimation becomes

$$
f(\mathbf{x})=\sum_{k=1}^{N} \alpha_{k} K\left(\mathbf{x}, \mathbf{x}_{k}\right)+b,
$$

where $\alpha_{k}$ and $b$ are the solutions to (34). The design values of $\gamma$ and $\sigma$ can be determined during the training of LS SVM. 
4.2. Input/Output Space Design of LS SVM. For the highly nonlinear input/output modeling problem discussed here, the LS SVM algorithm can provide an effective and viable solution. Besides, for land vehicle applications, the horizontal positioning performance is generally the main concern, as presented in a large body of literature $[4,9,10,12,13]$. Therefore, to simplify the LS VSM network structure and improve computational efficiency, the latitudinal and longitudinal components of the difference between the position error estimated after the measurement update 1 and that estimated after sequential measurement updates 1 and 2 will be selected as the outputs of LS SVM; that is,

$$
\text { Outputs }_{\mathrm{LSSVM}}=\left[\begin{array}{ll}
\delta \widetilde{L} & \delta \tilde{\lambda}
\end{array}\right],
$$

where $\delta \widetilde{L}=\mathbf{X}_{1}[1]-\mathbf{X}_{2}[1]$ is the latitudinal component and $\delta \tilde{\lambda}=\mathbf{X}_{1}[2]-\mathbf{X}_{2}[2]$ is the longitudinal component. $\mathbf{X}_{1}[1]$ and $\mathbf{X}_{1}[2]$ denote the first and second elements of the state vector $\mathbf{X}_{1}$, respectively, while $\mathbf{X}_{2}[1]$ and $\mathbf{X}_{2}[2]$ are the first and second elements of the state vector $\mathbf{X}_{2}$, respectively. As discussed above, $\mathbf{X}_{i}$ is the estimated state vector after the $i$ th measurement update $(i=1,2)$.

Also for simplifying the LS VSM structure, only the parameters that are the main factors affecting the outputs of LS SVM will be selected as its inputs. According to the mechanism of the inertial navigation, the fundamental error sources affecting the outputs (38) mainly include the measurement errors of IMU sensors, especially the longitudinal and lateral accelerometers and the yaw gyro and the time since the GPS signal is lost. Therefore, the inputs of LS SVM here are chosen as

$$
\text { Inputs }_{\mathrm{LSSVM}}=\left[\begin{array}{llll}
f_{x} & f_{y} & \omega_{z} & T
\end{array}\right]
$$

where $f_{x}, f_{y}$, and $\omega_{z}$ are the longitudinal and lateral accelerations and the yaw rate, respectively, which are output by IMU. $T$ denotes the time elapsed since the GPS signal is lost. From (39), it can be found that the adopted inputs have the advantage that they are all easy to determine.

\section{Field Tests and Results}

To evaluate the performance of the hybrid intelligent multisensor positioning methodology proposed in this paper, field tests were conducted on a Buick Sail SRV vehicle. It was equipped with a low-cost NovAtel Superstar II GPS receiver with $1 \mathrm{~Hz}$ update rate, Crossbow MEMS-based IMU VG440CA-200 inertial sensors sampled at $100 \mathrm{~Hz}$, and SDI electronic compass that provides the yaw angle measurement at $1 \mathrm{~Hz}$. The low-cost IMU VG440CA-200 adopted here includes three gyroscopes and three accelerometers, which are all made based on MEMS technology. The sensor accuracies $(1 \sigma)$ are $0.05 \mathrm{~m} / \mathrm{s}$ and $3 \mathrm{~m}$ for the GPS velocity and position when its signal is available and $0.15^{\circ}$ for the yaw angle of electronic compass. The field tests were carried out along a representative route in an open environment, as shown in Figure 2. Since GPS signals were available all the time, the SINS/GPS/Electronic Compass/Velocity Constraint integrated solution based on KF can be obtained and utilized

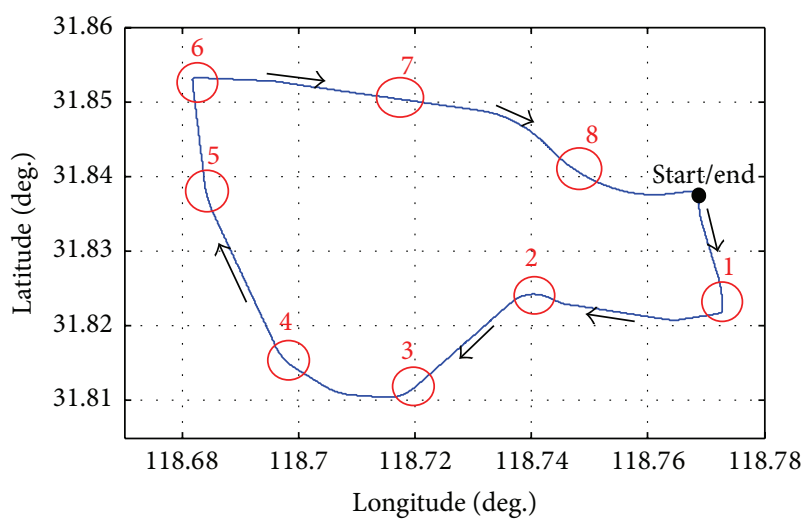

FIGURE 2: Field test trajectory with 8 GPS outages indicated.

as a reference for performance evaluation. During the tests, all sensor data were recorded and then the positioning methods were evaluated using the logged data.

Figure 2 shows the trajectory of this selected test route, which includes typical driving conditions such as straight motion, curved driving, and $90^{\circ}$ turns. Throughout the whole test, a sufficient number of satellites were available. To cover different driving conditions and dynamics and assess the positioning performance comprehensively, eight simulated $40 \mathrm{~s}$ GPS signal outages are intentionally introduced at eight typical locations along the trajectory, which are also marked in numeric labels and shown in Figure 2. The position error discussed in the following denotes the horizontal Euclidean distance error between the estimated position and the corresponding reference, which is the main concern for land vehicle positioning.

\subsection{Performance Comparison between KF and Proposed} Method. Table 1 gives a quantitative comparison of the RMS and maximum position errors between the traditional KF method and the proposed method for the simulated eight 40 s GPS outages. From Table 1, it is clear that the proposed hybrid positioning method outperforms the KF and offers much better accuracy for all GPS outages, except for outage 4. For outage 4, the maximum position error of KF is $17.97 \mathrm{~m}$, which is better than that of the proposed method, that is, $21.18 \mathrm{~m}$, but the difference between them is small and only about $3 \mathrm{~m}$. Besides, as far as the RMS value of the position error is concerned, the proposed method is superior to the KF, which achieves $38.1 \%$ improvement in accuracy during this GPS outage. For the remaining seven outages, the proposed method achieves the significant improvement in the positioning accuracy over the KF method. The proposed method can maintain the maximum position errors within the range of $5 \sim 22 \mathrm{~m}$ (the RMS range is $3 \sim 11 \mathrm{~m}$ ), as opposed to the range of 17 59 $\mathrm{m}$ (the RMS range is 12 26 $\mathrm{m}$ ) achieved by the traditional KF. For the remaining seven outages, the average improvement is more than $56 \%$ whether for the RMS error or for the maximum error.

To further illustrate the improvements of the proposed method over the traditional KF method, three representative GPS outages, that is, 1,5 , and 8 , are chosen to show their 
TABLE 1: Statistics of position errors for KF and proposed method.

\begin{tabular}{|c|c|c|c|c|c|c|}
\hline \multirow{2}{*}{ Outage number } & \multicolumn{3}{|c|}{ RMS } & \multicolumn{3}{|c|}{ Maximum } \\
\hline & $\mathrm{KF}$ & Proposed & Improvement (\%) & $\mathrm{KF}$ & Proposed & Improvement (\%) \\
\hline 1 & 19.56 & 8.37 & 57.2 & 28.54 & 14.77 & 48.3 \\
\hline 2 & 17.95 & 6.87 & 61.7 & 35.15 & 13.99 & 60.2 \\
\hline 3 & 13.93 & 8.67 & 37.8 & 28.27 & 19.59 & 30.7 \\
\hline 4 & 12.58 & 7.79 & 38.1 & 17.97 & 21.18 & - \\
\hline 5 & 18.84 & 3.81 & 79.8 & 36.96 & 5.77 & 84.4 \\
\hline 6 & 17.75 & 10.46 & 41.1 & 31.43 & 19.87 & 36.8 \\
\hline 7 & 25.97 & 8.70 & 66.5 & 58.05 & 15.01 & 74.1 \\
\hline 8 & 15.01 & 5.51 & 63.3 & 31.22 & 13.11 & 58.0 \\
\hline
\end{tabular}



FIGURE 3: Positioning results during GPS outage 1.

trajectories, as illustrated in Figures 3-5. Figure 3 shows their positioning results for GPS outage 1, which is introduced in a $90^{\circ}$ turn where the driving maneuvers change greatly. During this GPS outage, the maximum value of the position error of traditional $\mathrm{KF}$ is $28.54 \mathrm{~m}$, whereas that of the proposed method is reduced to $14.77 \mathrm{~m}$, which means a $48.3 \%$ performance improvement. Figure 4 gives the positioning results for GPS outage 5, which is in a typical driving scenario where the vehicle goes through a straight-curved-straight road. The solution of the proposed method follows the true trajectory more closely and provides an approximately $80 \%$ better accuracy than the traditional KF for both the RMS and maximum values of the position error. Figure 5 shows the positioning results for GPS outage 8, during which the vehicle goes through a continuously curved road. Similarly, the proposed method provides more accurate positioning outputs than the traditional KF, with about $60 \%$ performance improvement.

5.2. Performance Evaluation in Longer GPS Outage Conditions. Note that, in Section 5.1, we only discuss and analyze

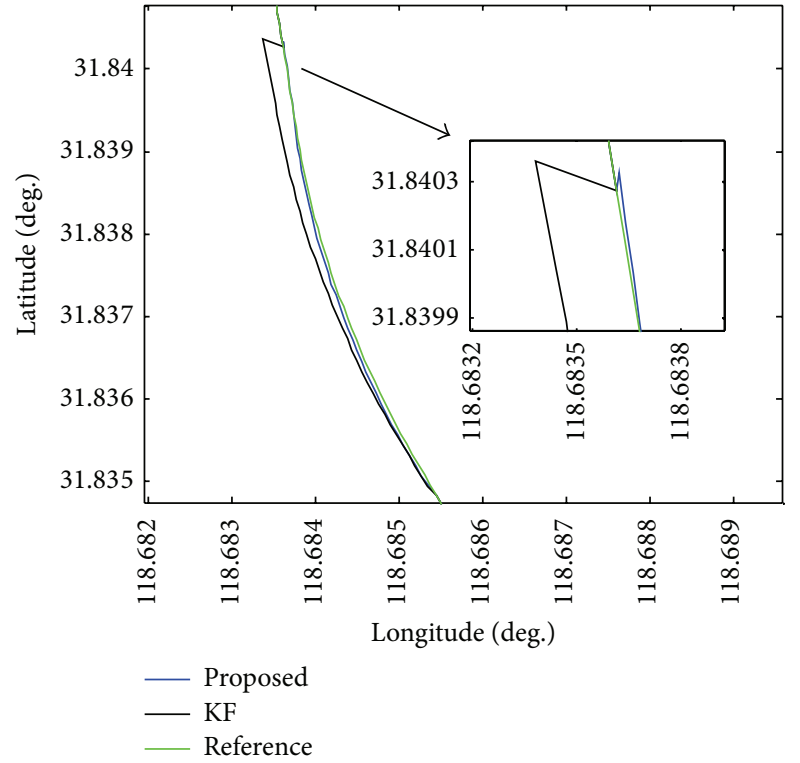

FIGURE 4: Positioning results during GPS outage 5.

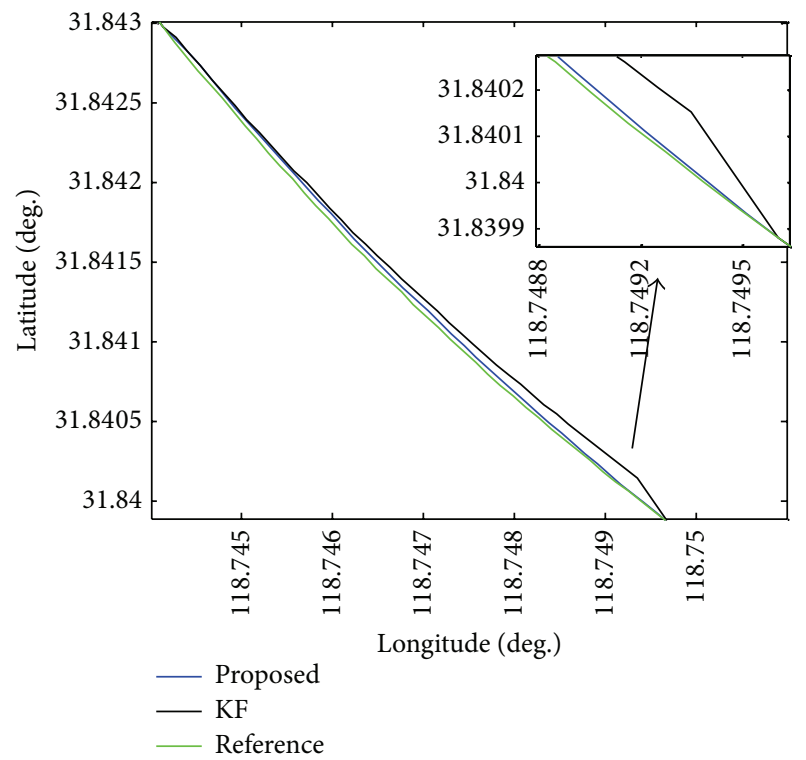

FIGURE 5: Positioning results during GPS outage 8. 


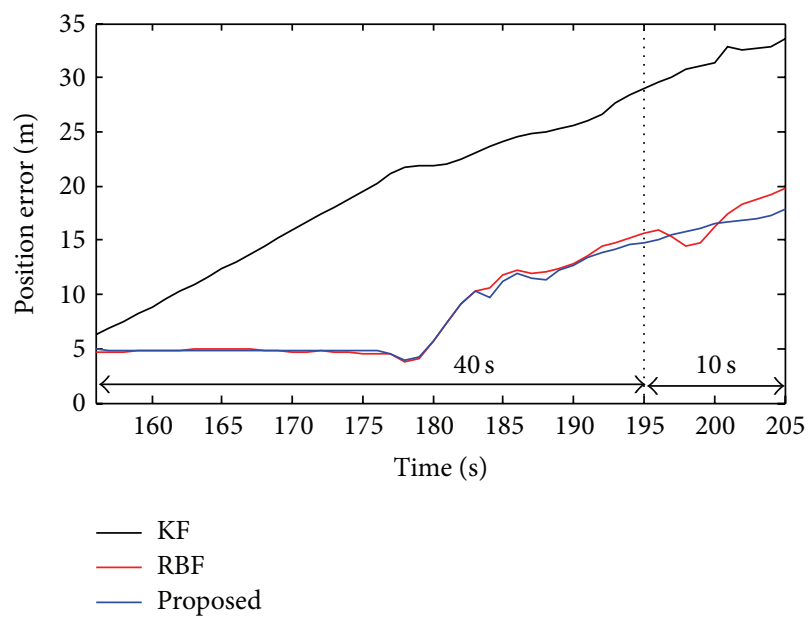

(a)

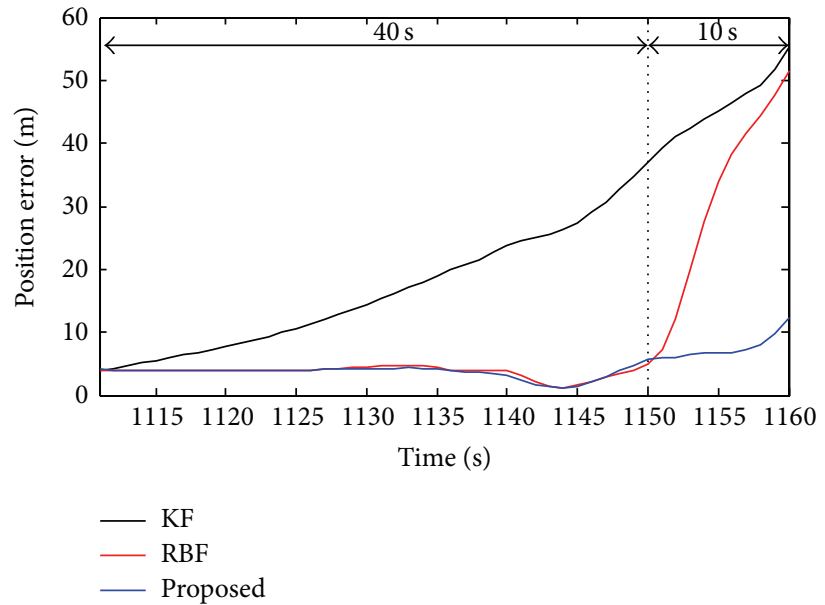

(b)

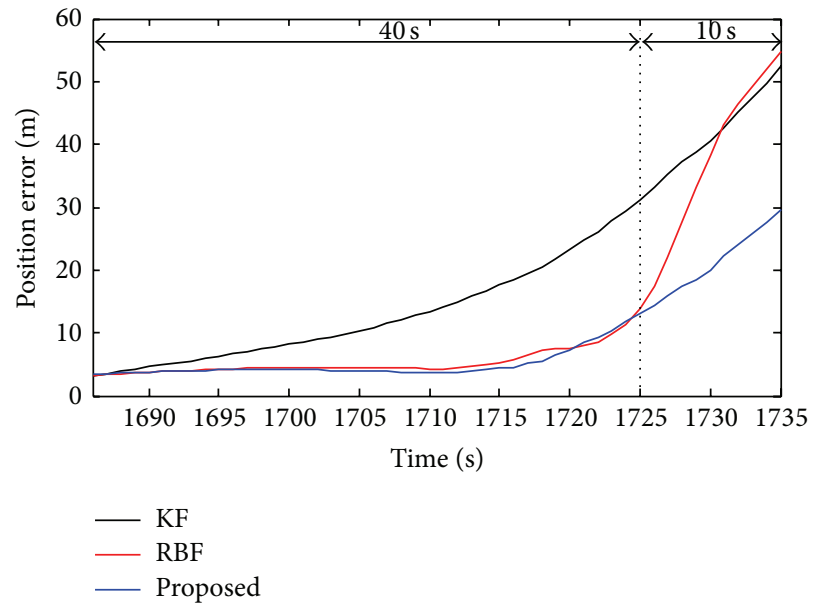

(c)

FIGURE 6: Position errors for three GPS outages (extended to $50 \mathrm{~s}$ ): (a) during outage 1; (b) during outage 5; (c) during outage 8.

the positioning performance for the 40 s GPS outage duration, which is equal to the designed sliding window size (i.e., $40 \mathrm{~s}$ ). However, in practice, the period of GPS outage cannot be determined in advance. Therefore, the positioning performance should also be evaluated in conditions where the GPS outage time exceeds the size of the window for training to verify the generalization ability of the positioning system. Such evaluation is seldom discussed in the existing literature.

To assess the performance in longer GPS outage conditions, the size of the sliding window for training is kept unchanged but the GPS outage duration is extended from $40 \mathrm{~s}$ to $50 \mathrm{~s}$. Besides, to compare with traditional neural network methods, the radial basis function (RBF) network as utilized in much literature is also designed. Specifically, the RBF method here denotes that the LS SVM module in Figure 1 is replaced by the RBF network with the same inputs/outputs and other modules in Figure 1 are kept unchanged. The model parameters of RBF are determined mainly via trial to achieve high training accuracy and good computational efficiency, which include 0.01 for the MSE (mean squared error) goal, 3 for the spread of radial basis functions, 40 for the maximum number of neurons, and 25 for the number of neurons to add between displays. Three typical GPS outages above, that is, 1 , 5 , and 8 , are still chosen to be analyzed. Figure 6 shows the position errors for three extended GPS outages. Figures 79 illustrate the positioning results for these three extended GPS outages (i.e., 1, 5, and 8), respectively. In Figures 8-9, the locally enlarged plots are also shown.

From Figure 6, both the proposed and RBF methods can significantly enhance the positioning accuracy compared with the traditional KF when the GPS outage is less than $40 \mathrm{~s}$. Moreover, they exhibit similar positioning performance within $40 \mathrm{~s}$ GPS outage. However, once the GPS outage is longer than $40 \mathrm{~s}$, the prediction stability of RBF deteriorates, while that of the proposed method is still good and reliable, which is especially remarkable for outages 5 and 8 , as shown in Figures 6(b)-6(c), 8, and 9. That is, the generalization performance of the proposed method is obviously superior to that of traditional RBF method. The main reason is that the overfitting problem easily occurs for the RBF, which cannot ensure the generalization ability of $\mathrm{RBF}$ once the prediction 


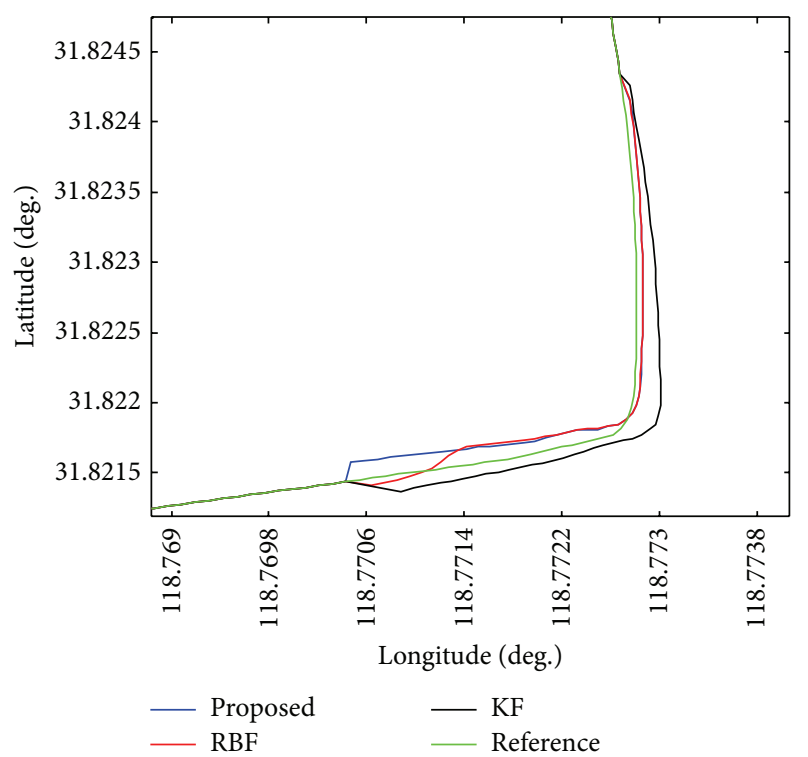

FIgURE 7: Positioning results during GPS outage 1 (extended to $50 \mathrm{~s}$ ).

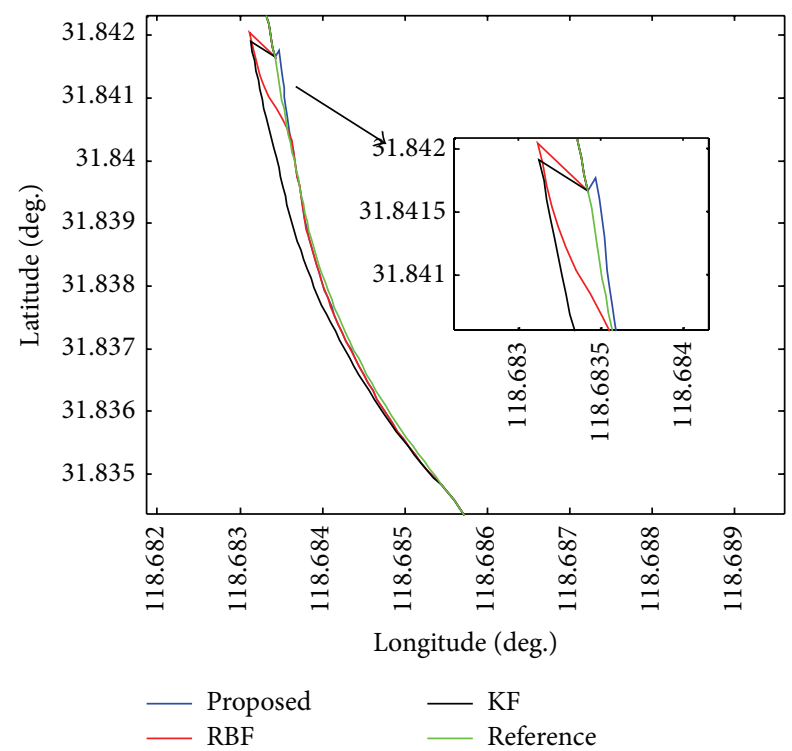

FIGURE 8: Positioning results during GPS outage 5 (extended to $50 \mathrm{~s}$ ).

data is beyond the space covered by the training sample. For example, for the extended GPS outage 5, the maximum value of the position error of RBF reaches $51.42 \mathrm{~m}$ as indicated in Figure 6(b), which is very close to that of KF, that is, $55.40 \mathrm{~m}$. In contrast, the maximum value of the position error of the proposed method is only $12.36 \mathrm{~m}$, which achieves an accuracy improvement of $76 \%$ and $78 \%$ over RBF and KF, respectively. Figure 8 clearly shows that the proposed method can provide more accurate positioning solution, which is much closer to the true trajectory.

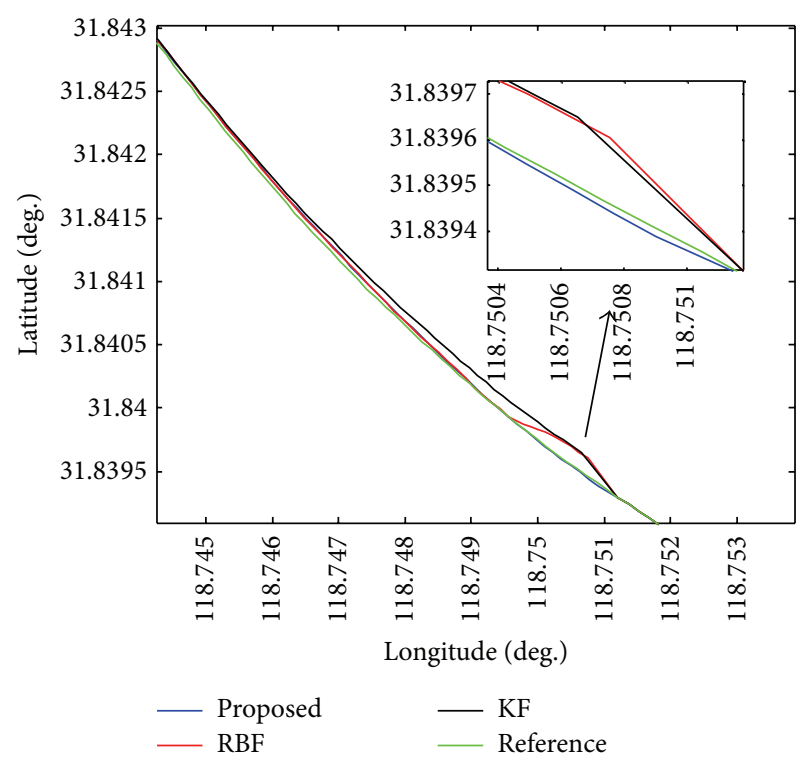

FIGURE 9: Positioning results during GPS outage 8 (extended to $50 \mathrm{~s})$.

\section{Conclusions}

To achieve reliable positioning performance even in adverse GPS environments, a hybrid intelligent multisensor positioning methodology is proposed for land vehicles. The proposed method can fuse the information from low-cost sensors including GPS, MEMS-based SINS, electronic compass, and lateral velocity constraint. For such information, the improved KF with sequential measurement-update processing is first developed to realize the filtering fusion. Further, the LS SVM-based intelligent module is designed and augmented with the improved KF to form the hybrid positioning system. When GPS works well, the LS SVM module is trained and updated using the recent input/output data. Once GPS outage occurs, it switches to predict the position error to achieve more accurate positioning performance.

The field tests on typical roads have been performed for evaluation of the proposed methodology. The experimental results verify that the proposed method can significantly improve the positioning accuracy over the traditional KF method for most conditions under GPS outages. In addition, it has shown much stronger prediction stability than other AI-based methods such as RBF, which indicates that it can achieve more satisfactory generalization ability.

\section{Appendix}

Consider

$$
\mathbf{F}=\left[\begin{array}{ccccc}
\mathbf{F}_{P P} & \mathbf{F}_{P V} & \mathbf{F}_{P \psi} & \mathbf{0}_{3 \times 3} & \mathbf{0}_{3 \times 3} \\
\mathbf{F}_{V P} & \mathbf{F}_{V V} & \mathbf{F}_{V \psi} & \mathbf{C}_{b}^{n} & \mathbf{0}_{3 \times 3} \\
\mathbf{F}_{\psi P} & \mathbf{F}_{\psi V} & \mathbf{F}_{\psi \psi} & \mathbf{0}_{3 \times 3} & -\mathbf{C}_{b}^{n} \\
\mathbf{0}_{6 \times 3} & \mathbf{0}_{6 \times 3} & \mathbf{0}_{6 \times 3} & \mathbf{0}_{6 \times 3} & \mathbf{0}_{6 \times 3}
\end{array}\right],
$$


where all the subcomponents in $\mathbf{F}$ are discussed in detail in [19]. For instance,

$$
\begin{aligned}
& \mathbf{F}_{\psi P}=\left[\begin{array}{ccc}
0 & 0 & \frac{V_{N}}{\left(R_{n}+h\right)^{2}} \\
-\omega_{i e} \sin L & 0 & \frac{-V_{E}}{\left(R_{e}+h\right)^{2}} \\
\omega_{i e} \cos L+\frac{V_{E} \sec ^{2} L}{R_{e}+h} & 0 & \frac{-V_{E} \tan L}{\left(R_{e}+h\right)^{2}}
\end{array}\right], \\
& \mathbf{F}_{\psi V}=\left[\begin{array}{ccc}
0 & \frac{-1}{R_{n}+h} & 0 \\
\frac{1}{R_{e}+h} & 0 & 0 \\
\frac{\tan L}{R_{e}+h} & 0 & 0
\end{array}\right] \\
& \mathbf{F}_{\psi \psi}=\left[\begin{array}{ccc}
0 & \omega_{i e} \sin L+\frac{V_{E} \tan L}{R_{e}+h} & -\left(\omega_{i e} \cos L+\frac{V_{E}}{R_{e}+h}\right) \\
-\left(\omega_{i e} \sin L+\frac{V_{E} \tan L}{R_{e}+h}\right) & 0 & -\frac{V_{N}}{R_{n}+h} \\
\omega_{i e} \cos L+\frac{V_{E}}{R_{e}+h} & \frac{V_{N}}{R_{n}+h}
\end{array}\right] \text {, }
\end{aligned}
$$

where $R_{n}=a\left(1-2 f+3 f \sin ^{2} L\right), R_{e}=a\left(1+f \sin ^{2} L\right) \cdot a$ is the Earth semimajor axis length, and $f$ is the Earth ellipsoid oblateness. $\omega_{i e}$ is the Earth rotation rate. $L$ is the latitude of the vehicle location, and $h$ is the altitude of the vehicle location. $V_{E}$ and $V_{N}$ are the east and north velocity components, respectively. One has

$$
\begin{aligned}
\mathbf{G} & =\left[\begin{array}{cc}
\mathbf{0}_{3 \times 3} & \mathbf{0}_{3 \times 3} \\
\mathbf{C}_{b}^{n} & \mathbf{0}_{3 \times 3} \\
\mathbf{0}_{3 \times 3} & -\mathbf{C}_{b}^{n} \\
\mathbf{0}_{6 \times 3} & \mathbf{0}_{6 \times 3}
\end{array}\right] \\
\mathbf{W} & =\left[\begin{array}{llllll}
w_{a x} & w_{a y} & w_{a z} & w_{g x} & w_{g y} & w_{g z}
\end{array}\right],
\end{aligned}
$$

where $w_{a x}, w_{a y}$, and $w_{a z}$ denote three accelerometer white noises, while $w_{g x}, w_{g y}$, and $w_{g z}$ are three gyro white noises. One has

$$
\Phi(k+1, k) \approx \mathbf{I}+\mathbf{F}\left(t_{k}\right)\left(t_{k+1}-t_{k}\right),
$$

where $t_{k+1}$ and $t_{k}$ are the discrete time. Consider

$$
\begin{aligned}
\Gamma(k) & \approx \mathbf{G}\left(t_{k}\right)\left(t_{k+1}-t_{k}\right) \\
\mathbf{W}(k) & =\frac{1}{t_{k+1}-t_{k}} \int_{t_{k}}^{t_{k+1}} \mathbf{W}(t) d t .
\end{aligned}
$$

\section{Conflict of Interests}

The authors declare that there is no conflict of interests regarding the publication of this paper.

\section{Acknowledgments}

This work was supported by the National Natural Science Foundation of China (Grant no. 61273236), the Jiangsu Provincial Basic Research Program (Natural Science Foundation, Grant no. BK2010239), and the Doctoral Fund for Youth Teachers of Ministry of Education of China (no. 200802861061).

\section{References}

[1] N. R. Velaga, M. A. Quddus, A. L. Bristow, and Y. Zheng, "Mapaided integrity monitoring of a land vehicle navigation system," IEEE Transactions on Intelligent Transportation Systems, vol. 13, no. 2, pp. 848-858, 2012.

[2] I. Skog and P. Händel, "In-car positioning and navigation technologiesa-a survey," IEEE Transactions on Intelligent Transportation Systems, vol. 10, no. 1, pp. 4-21, 2009.

[3] H. Liu, S. Nassar, and N. El-Sheimy, "Two-filter smoothing for accurate INS/GPS land-vehicle navigation in urban centers," IEEE Transactions on Vehicular Technology, vol. 59, no. 9, pp. 4256-4267, 2010.

[4] J. Huang and H.-S. Tan, "A low-order DGPS-based vehicle positioning system under urban environment," IEEE/ASME Transactions on Mechatronics, vol. 11, no. 5, pp. 567-575, 2006.

[5] Z. Wu, M. Yao, H. Ma, and W. Jia, "Improving accuracy of the vehicle attitude estimation for low-cost INS/GPS integration aided by the GPS-measured course angle," IEEE Transactions on Intelligent Transportation Systems, vol. 14, no. 2, pp. 553-564, 2013. 
[6] F. Caron, E. Duflos, D. Pomorski, and P. Vanheeghe, "GPS/IMU data fusion using multisensor Kalman filtering: introduction of contextual aspects," Information Fusion, vol. 7, no. 2, pp. 221230, 2006.

[7] M. Cossaboom, J. Georgy, T. Karamat, and A. Noureldin, "Augmented Kalman filter and map matching for 3D RISS/GPS integration for land vehicles," International Journal of Navigation and Observation, vol. 2012, Article ID 576807, 16 pages, 2012.

[8] L. Semeniuk and A. Noureldin, "Bridging GPS outages using neural network estimates of INS position and velocity errors," Measurement Science and Technology, vol. 17, no. 10, pp. 27832798, 2006.

[9] R. Toledo-Moreo, M. A. Zamora-Izquierdo, B. Úbeda-Miñarro, and A. F. Gómez-Skarmeta, "High-integrity IMM-EKF-based road vehicle navigation with low-cost GPS/SBAS/INS," IEEE Transactions on Intelligent Transportation Systems, vol. 8, no. 3, pp. 491-511, 2007.

[10] A. Noureldin, T. B. Karamat, M. D. Eberts, and A. El-Shafie, "Performance enhancement of MEMS-based INS/GPS integration for low-cost navigation applications," IEEE Transactions on Vehicular Technology, vol. 58, no. 3, pp. 1077-1096, 2009.

[11] F. Jiancheng and Y. Sheng, "Study on innovation adaptive EKF for in-flight alignment of airborne POS," IEEE Transactions on Instrumentation and Measurement, vol. 60, no. 4, pp. 1378-1388, 2011.

[12] S. Adusumilli, D. Bhatt, H. Wang, P. Bhattacharya, and V. Devabhaktuni, "A low-cost INS/GPS integration methodology based on random forest regression," Expert Systems with Applications, vol. 40, no. 11, pp. 4653-4659, 2013.

[13] N. El-Sheimy, K.-W. Chiang, and A. Noureldin, “The utilization of artificial neural networks for multisensor system integration in navigation and positioning instruments," IEEE Transactions on Instrumentation and Measurement, vol. 55, no. 5, pp. 1606$1615,2006$.

[14] W. Abdel-Hamid, A. Noureldin, and N. El-Sheimy, "Adaptive fuzzy prediction of low-cost inertial-based positioning errors," IEEE Transactions on Fuzzy Systems, vol. 15, no. 3, pp. 519-529, 2007.

[15] K. Saadeddin, M. F. Abdel-Hafez, M. A. Jaradat, and M. A. Jarrah, "Optimization of intelligent-based approach for low-cost INS/GPS navigation system," in Proceedings of the International Conference on Unmanned Aircraft Systems (ICUAS '13), pp. 668677, Atlanta, Ga, USA, May 2013.

[16] X. Lei and J. Li, "An adaptive navigation method for a small unmanned aerial rotorcraft under complex environment," Measurement, vol. 46, no. 10, pp. 4166-4171, 2013.

[17] R. Sharaf and A. Noureldin, "Sensor integration for satellitebased vehicular navigation using neural networks," IEEE Transactions on Neural Networks, vol. 18, no. 2, pp. 589-594, 2007.

[18] R. Sharaf, A. Noureldin, A. Osman, and N. El-Sheimy, "Online INS/GPS integration with a radial basis function neural network," IEEE Aerospace and Electronic Systems Magazine, vol. 20, no. 3, pp. 8-14, 2005.

[19] J. Farrell and M. Barth, The Global Positioning System and Inertial Navigation, McGraw-Hill, New York, NY, USA, 1999.

[20] J. A. Farrell, T. D. Givargis, and M. J. Barth, "Real-time differential carrier phase GPS-aided INS," IEEE Transactions on Control Systems Technology, vol. 8, no. 4, pp. 709-721, 2000.

[21] X. Li and W. Zhang, "An adaptive fault-tolerant multisensor navigation strategy for automated vehicles," IEEE Transactions on Vehicular Technology, vol. 59, no. 6, pp. 2815-2829, 2010.
[22] S. Godha and M. E. Cannon, "GPS/MEMS INS integrated system for navigation in urban areas," GPS Solutions, vol. 11, no. 3, pp. 193-203, 2007.

[23] Y. C. Yang and J. A. Farrell, "Magnetometer and differential carrier phase GPS-aided INS for advanced vehicle control," IEEE Transactions on Robotics and Automation, vol. 19, no. 2, pp. 269-282, 2003.

[24] X. Li, X. Song, and C. Chan, "Reliable vehicle sideslip angle fusion estimation using low-cost sensors," Measurement, vol. 51, no. 1, pp. 241-258, 2014.

[25] P. Samui, "Application of least square support vector machine (LSSVM) for determination of evaporation losses in reservoirs," Engineering, vol. 3, no. 4, pp. 431-434, 2011.

[26] H.-Q. Wang, F.-C. Sun, Y.-N. Cai, L.-G. Ding, and N. Chen, "An unbiased LSSVM model for classification and regression," Soft Computing, vol. 14, no. 2, pp. 171-180, 2010.

[27] T. Hacib, Y. Le Bihan, M. K. Smail, M. R. Mekideche, O. Meyer, and L. Pichon, "Microwave characterization using ridge polynomial neural networks and least-square support vector machines," IEEE Transactions on Magnetics, vol. 47, no. 5, pp. 990-993, 2011.

[28] C. Yan-Gao and M. Guangwen, "Electricity price forecasting based on support vector machine trained by genetic algorithm," in Proceedings of the 3rd International Symposium on Intelligent Information Technology Application (IITA '09), pp. 292-295, Nanchang, China, November 2009. 


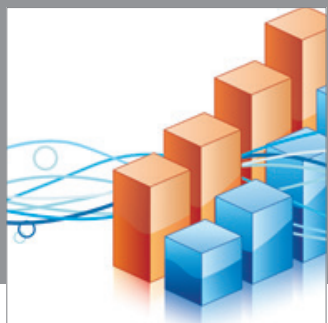

Advances in

Operations Research

mansans

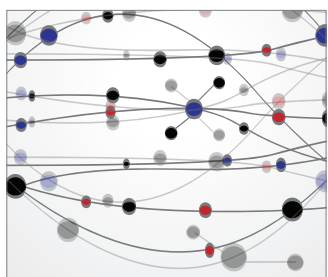

The Scientific World Journal

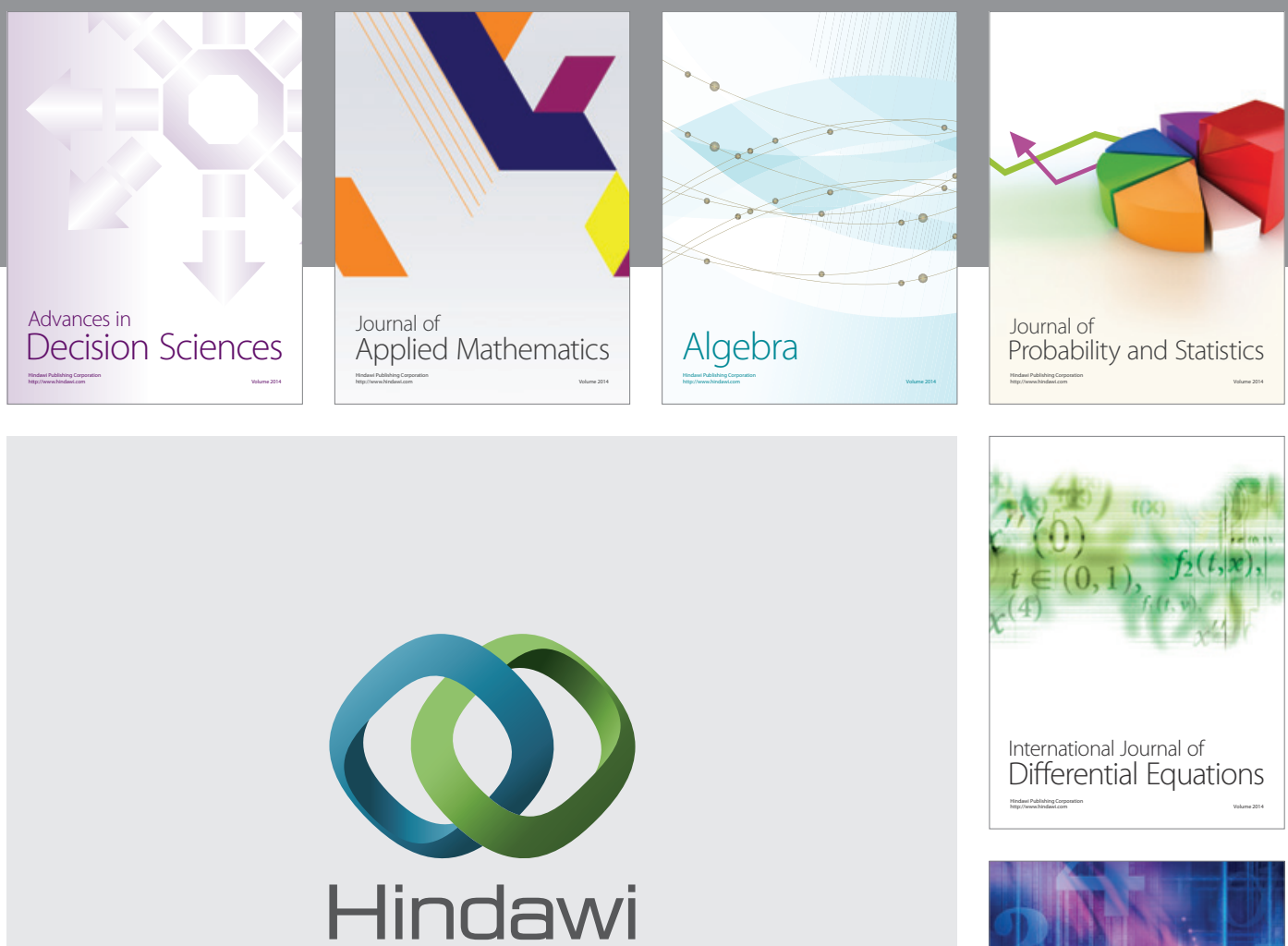

Submit your manuscripts at http://www.hindawi.com
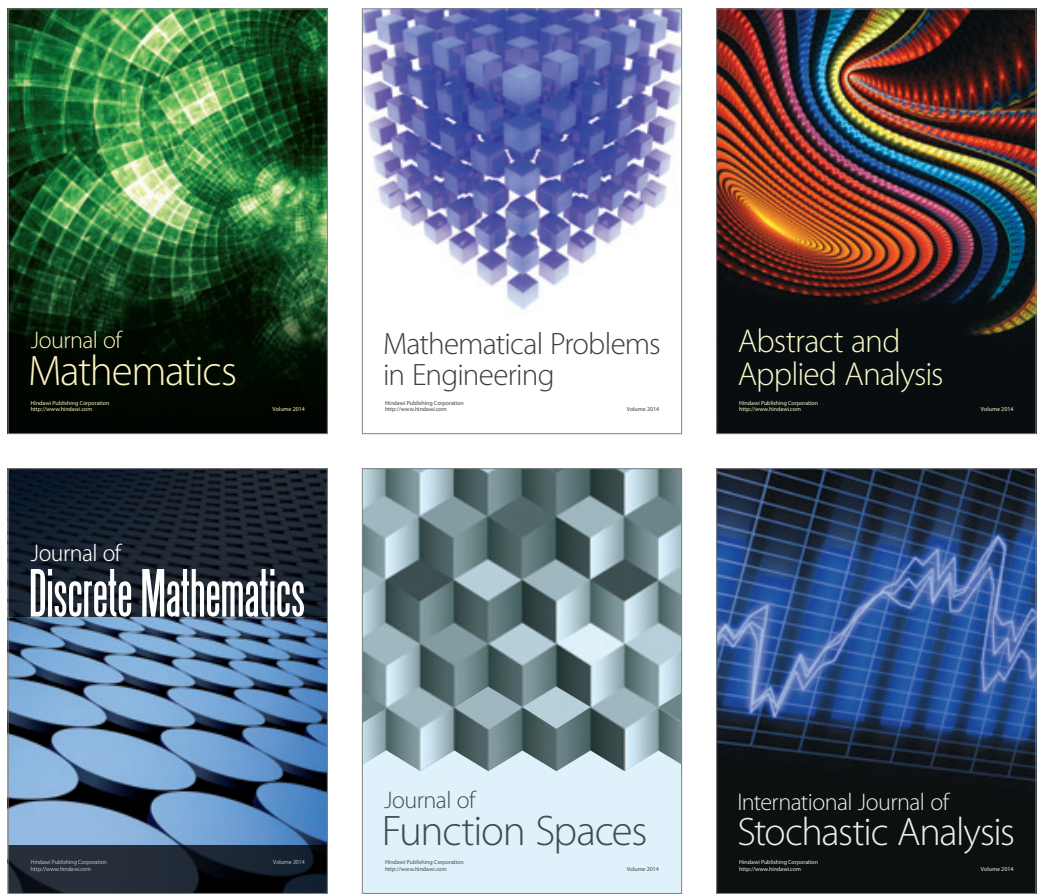

Journal of

Function Spaces

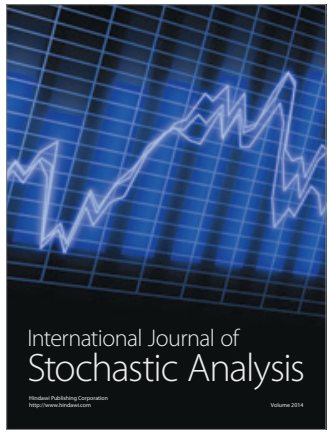


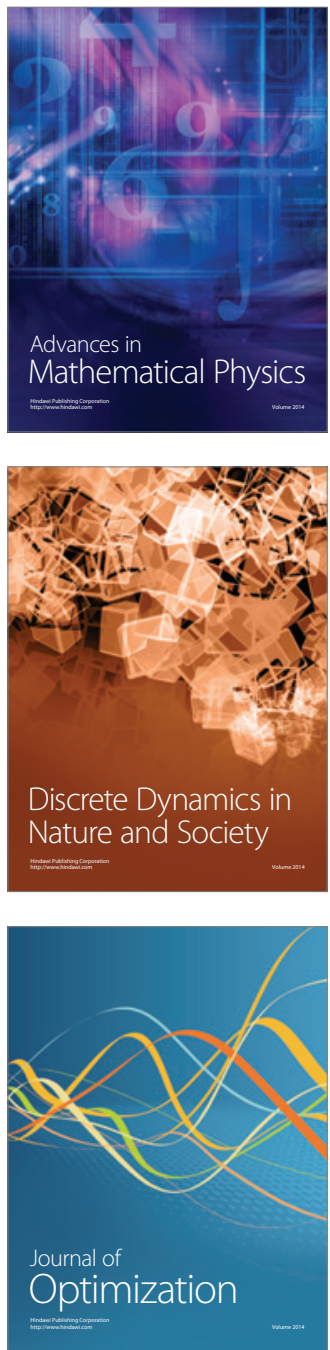